\title{
Diagnostic value of the balloon expulsion test compared with anorectal manometry in Indian patients with dyssynergic defecation
}

\author{
Mayank Jain ${ }^{1}$, Saransh Singh ${ }^{2}$, Rajiv Baijal ${ }^{2}$ \\ ${ }^{1}$ Department of Gastroenterology, Arihant Hospital and Research Centre, Indore, India \\ 2Department of Gastroenterology, Pushpavati Singhania Hospital and Research Centre, New Delhi, India
}

Gastroenterology Rev 2020; 15 (2): 151-155

DOI: https://doi.org/10.5114/pg.2020.95558

Key words: dyssynergia, manometry, balloon expulsion.

Address for correspondence: Dr. Mayank Jain, Department of Gastroenterology, Arihant Hospital and Research Centre, Indore, India, phone: +91 7312365688, e-mail: mayank4670@rediffmail.com

\begin{abstract}
Introduction: Digital rectal examination (DRE) and balloon expulsion test (BET) are simple tests to diagnose dyssynergic defecation (DD).

Aim: To determine differences in symptoms and manometry findings in patients with abnormal BET and normal BET. The secondary objective was to ascertain the sensitivity and specificity of BET and DRE + BET for the diagnosis of DD in an Indian setting using ARM findings as the gold standard.

Material and methods: Retrospective analysis of patients with chronic constipation referred for anorectal manometry (ARM) between December 2012 and March 2019. DD was diagnosed using ARM. Findings on BET and, in a subset of cases, on DRE + BET were compared with ARM findings. The data were analyzed for sensitivity, specificity, positive predictive value (PPV), and negative predictive value (NPV). Agreement of BET and DRE + BET with ARM was calculated using Cohen's $\kappa$ coefficient. A $p$-value of $<0.05$ was considered significant.

Results: A total of 1006 cases (734 males, 73\%) formed the study cohort. Patients with abnormal BET more frequently reported digitation, bleeding per rectum, and straining $(p<0.00001)$. Moreover, they had a significantly higher median basal pressure compared to those with normal BET ( 80 vs. $67, p=0.03$ ). DD was significantly more common in those with abnormal BET. The sensitivity, specificity, PPV, and NPV of BET in detecting DD were $28.29 \%, 97.15 \%, 81.13 \%$, and $75.78 \%$, respectively. The percentage of agreement was $76.34 \%$, and there was fair degree of correlation between the two tests. In a smaller subset of cases (166), DRE and BET findings were both available for analysis. We noted that the sensitivity, specificity, PPV, and NPV of combined DRE + BET were $57.63 \%, 88.79 \%, 73.91 \%$, and $79.17 \%$, respectively. The Cohen's $\kappa$ correlation coefficient was 0.49 , suggesting moderate agreement.

Conclusions: Patients with abnormal BET more frequently report digitation, straining, and bleeding per rectum, and have higher resting anal pressure. BET is a good screening test for DD in an Indian setting.
\end{abstract}

\section{Introduction}

Dyssynergic defecation (DD) is commonly diagnosed in the Indian setting using anorectal manometry (ARM) with or without defecography. An earlier study highlighted the importance of digital rectal examination (DRE) as an effective screening tool for DD in an Indian setting [1]. Apart from DRE, balloon expulsion test (BET) is also an inexpensive and simple procedure used clinically to diagnose DD.

BET was first described by Preston and LennardJones [2]. However, to date there is no consensus on the methodology of the test. Experts the world over either use air-filled or water-filled balloons in a lying or seated position to perform BET. Recommended time values range from less than $1 \mathrm{~min}$ to up to $5 \mathrm{~min}$ in different studies [3].
Aim
The aim of the present study was to determine the differences in symptoms and ARM findings in patients with abnormal and normal BET. The secondary aim was to ascertain the sensitivity and specificity of BET for the 
diagnosis of DD in an Indian setting using ARM findings as the gold standard. Moreover, in a subset of our study group, we assessed the utility of DRE + BET for the diagnosis of DD.

\section{Material and methods}

The present study is a retrospective analysis. It includes all patients with chronic constipation referred for ARM to the authors between December 2012 and March 2019. Patients included in the study were recruited from Arihant Hospital and Research Centre, Indore (MJ) and Pushpavati Singhania Hospital and Research Institute, New Delhi (RB). The protocol followed for evaluation of chronic constipation at both centres was the same. A standard assessment form was filled in for all patients. It included clinical history, stool form and consistency as per Bristol Stool chart, and clinical classification, classified as irritable bowel syndrome with constipation (IBS-C) and functional constipation (FC). All patients had a digital rectal examination, sigmoidoscopy, or colonoscopy, in order to screen for solitary rectal ulcer syndrome (SRUS), haemorrhoids, fissure, and mechanical obstruction, and an ARM was performed. Colonic transit study and defecography was done only in a few cases (42) and hence was not included in the analysis.

Inclusion criteria: age > 18 years, symptom duration $>6$ months, no malignant lesions on colonoscopy.

Exclusion criteria: patients who were referred for evaluation for causes other than constipation like incontinence and post-surgical cases, colorectal cancers, age $<18$ years.

All patients were prepared using laxatives the night before the procedure. For patients with acute fissures or painful haemorrhoids, symptomatic treatment was done initially. They underwent ARM when they were pain free.

ARM was performed in the left lateral position with the hips flexed. A sixteen-channel silicone-rubber water perfusion manometry assembly (Ready Stock, Australia) was used. Data were recorded at $25 \mathrm{~Hz}$ and analysed using Trace Version 1.3v (Hebbard, Melbourne, Australia). The parameters included the following: anorectal pressures at rest (60 s), squeeze pressures (three attempts for a maximum duration of $20 \mathrm{~s}$ each), rectoanal inhibitory reflex, and rectal sensations. Rectal sensory testing was performed by inflating an intra-rectal balloon with progressively increasing volumes of air $(10 \mathrm{ml}$ increments; from 10 to $400 \mathrm{ml})$. The patients were asked to report regarding the first sensation, desire and urgency to defecate, and the maximum tolerable limit while the balloon was being inflated. Rectal hyposensitivity was defined as the maximum tolerable limit $>240 \mathrm{ml}$ or at least two of the following: (i) first sensation at volume $>25 \mathrm{ml}$, (ii) desire to defecate at volume $>150 \mathrm{ml}$, and (iii) urgency to defecate at volume $>200 \mathrm{ml}$ [4].

Recto-anal inhibitory reflex (RAIR) was also assessed during balloon inflation. It was considered present if there was a reduction in resting anal sphincter pressure on rectal balloon inflation.

BET was recorded after distending a rectal balloon with $50 \mathrm{ml}$ of air [5] and asking the patient to expel the balloon in the left lateral position [5, 6].

DD was diagnosed based on the following criteria:

1. Patients fulfilling the diagnostic criteria for functional constipation and/or constipation-predominant IBS as per Rome III definition.

2. Patients demonstrating dyssynergic pattern during repeated attempts to defecate - a dyssynergic pattern of defecation (types I-IV) was defined as a paradoxical increase in anal sphincter pressure (anal contraction), or less than $20 \%$ relaxation of the resting anal sphincter pressure, or inadequate propulsive forces observed with manometry.

BET was considered abnormal if the patient was unable to expel $50 \mathrm{ml}$ air from the inflated balloon within $1 \min [1]$.

In a subset of patients, DRE findings were also available. DRE was performed on the same day prior to the BET and ARM. DRE was done in the left lateral position with the hips flexed and using local anaesthetic jelly. Three key steps in DRE were: (a) palpation for tenderness, mass, stricture, and presence of stool; (b) resting anal tone assessment; and (c) squeeze evaluation for $30 \mathrm{~s}$ for intensity and sustainability. After this, the patient was asked to bear down. The examiner placed his left hand on the patient's abdomen to assess the pushing effort. The ability to relax the anal sphincter and perineal descent was noted. Dyssynergia on DRE was diagnosed if any two of the following findings were positive: the inability to contract the abdominal muscles, inability to relax the anal sphincter, a paradoxical contraction of the anal sphincter, or the absence of perineal descent $[1,7]$.

\section{Statistical analysis}

The data was collected and tabulated in a Microsoft Excel spreadsheet. Age and anorectal pressures were expressed as median and range. Variables like sex, symptom profile, co-morbid states, and the presence of DD were expressed as numbers and proportions. The data were analysed for sensitivity, specificity, positive predictive value (PPV), and negative predictive value (NPV). Agreement of BET and DRE + BET with ARM was 
calculated using Cohen's $\kappa$ coefficient. A $p$-value of $<0.05$ was considered significant.

\section{Results}

A total of 1120 patients underwent ARM during the study period. Of these, 114 were excluded based on the study criteria. Thus, 1006 cases (734 males, 73\%) formed the study cohort. The median age was 45 years (range: 18-82). The co-morbid states included diabetes mellitus in 151 (15\%), hypertension in 160 (16.1\%), coronary artery disease in 70 (6.95\%), and hypothyroidism in 23 (2.28\%). A total of 141 cases (14\%) had fissures/ haemorrhoids on colonoscopy examination. Nearly one third of cases (304, 30.2\%) had DD. Of these, 295 (97\%) cases had type 1 DD, 7 (2.3\%) had type II DD, and 1 each
(0.3\%) had type III and IV DD, respectively. Recto anal inhibitory reflex was present in $99.8 \%$ (1004) of cases. Rectal hyposensitivity was present in 625 (62.1\%) cases.

Table I shows the difference in the studied parameters among patients with abnormal and normal BET. Patients with abnormal BET more frequently reported digitation, bleeding per rectum, and straining $(p<0.00001)$. Moreover, they had a significantly higher median basal pressure compared to those with normal BET (80 vs. 67 , $p=0.03$ ). DD was significantly more common in those with abnormal BET.

As noted from Table II, sensitivity, specificity, PPV, and NPV of BET in detecting DD were $28.29 \%, 97.15 \%$, $81.13 \%$, and $75.78 \%$, respectively. The percentage of agreement was $76.34 \%$, and there was a fair degree of correlation between the two tests.

Table I. Comparison of symptoms and ARM parameters among patients with abnormal and normal BET

\begin{tabular}{lccc} 
Parameter & Abnormal BET $(n=106)$ & Normal BET $(n=900)$ & $P$-value \\
\hline Age, median (range) & $43(18-72)$ & $45(19-82)$ & 0.13 \\
\hline Sex: & & & 0.59 \\
\hline Male & $75(70.7 \%)$ & $659(73.3 \%)$ & $<0.00001$ \\
\hline Female & $31(29.3 \%)$ & $241(26.7 \%)$ & \\
\hline Symptoms: & $32(30.2 \%)$ & $42(4.7 \%)$ & $54(6 \%)$ \\
\hline Digitation & $56(52.8 \%)$ & $267(29.6 \%)$ & \\
\hline Straining & $31(29.2 \%)$ & $25(2.8 \%)$ & 0.03 \\
\hline Incomplete evacuation & $16(15 \%)$ & $67(43-113)$ & 0.24 \\
\hline Bleeding per rectum & & $109(80-175)$ & $<0.00001$ \\
\hline ARM parameters: & $80(57-112)$ & $218(24.2 \%)$ & \\
\hline Basal pressure [mm Hg] median (range) & $116(70-160)$ & & \\
\hline Squeeze pressures [mm Hg] median (range) & $86(81.1 \%)$ & & \\
\hline Presence of any form of dyssynergic defecation & & & \\
\hline
\end{tabular}

Table II. Sensitivity, specificity, NPV, and PPV of BET and DRE + BET in predicting DD

\begin{tabular}{llll}
\hline DD by ARM & Yes & No & Total \\
\hline Abnormal BET & 86 & 20 & 106 \\
\hline Normal BET & 218 & 682 & 900 \\
\hline
\end{tabular}

Sensitivity $28.29 \%$ (23.3-33.7\%), specificity $97.15 \%$ (95.63-98.25\%), NPV 75.78\% (74.4-77.0), PPV 81.13\% (72.93-87.28\%), percentage of agreement $76.34 \%$, Cohen's $\kappa=0.31$ (fair agreement)

\begin{tabular}{llll}
\hline DD by ARM & Yes & No & Total \\
\hline Abnormal BET + DRE & 34 & 12 & 46 \\
\hline Normal BET + DRE & 25 & 95 & 120 \\
\hline
\end{tabular}

Sensitivity 57.63\% (44.07-70.39\%), specificity 88.79\% (81.23-94.07\%), NPV 79.17\% (73.69-83.75\%), PPV 73.91\% (61.42-83.45\%), percentage of agreement $77.71 \%$, Cohen's $\kappa=0.49$ (moderate agreement) 
In a smaller subset of cases (166), DRE and BET findings were both available for analysis. We noted that the sensitivity, specificity, PPV, and NPV of combined DRE + BET were $57.63 \%, 88.79 \%, 73.91 \%$, and $79.17 \%$, respectively. The Cohen's $\kappa$ correlation coefficient was 0.49 suggesting moderate agreement.

\section{Discussion}

The present study highlights that patients with $a b$ normal BET more frequently report digitation, straining, and bleeding per rectum. The resting anal pressure is higher in these patients. With a high specificity and PPV, BET seems to be a good screening test for DD in an Indian setting. The combination of BET with DRE seems to correlate better with the diagnosis of DD on ARM than BET alone.

Cost effectiveness in health care is a major challenge in current clinical practice in India. Thus, it is important to devise a cheaper but satisfactory diagnostic approach for DD. We previously reported that the sensitivity, specificity, PPV, and NPV of DRE in the detection of DD was $69.7 \%, 81.5 \%, 82.1 \%$, and $68.75 \%$, respectively [1]. These results were similar to results from other researchers $[7,8]$.

In the present study, we determined the utility of BET for diagnosis of DD. As highlighted earlier, the BET technique is not standardised. A recent meta-analysis noted that 12 studies on BET described the use of the seated position because it better resembles the act of defecation [9]. The majority of the studies used water-filled BET [9] while a few used air-filled balloons [10-12]. Moreover, some studies used variable volumes ranging from 10 to $60 \mathrm{ml}$ for BET $[13,14]$. Differences in the material of the balloon used [3] and time given for the patient to expel the balloon $[10,15,16]$ are also likely to influence the BET. Chronic constipation is classified as FC and IBS-C. The crucial pathophysiological difference between the two is the difference in the degree of visceral sensitivities. Rectal hyposensitivity is commoner in FC $[17,18]$. It has been suggested that rectal hyposensitivity and absence of RAIR, both suggestive of autonomic neuropathy, may play a role in $12 \%$ to $30 \%$ of patients with chronic constipation $[19,20]$. Rectal hyposensitivity has been reported to be present in $50 \%$ to $60 \%$ of patients with chronic constipation [21]. In our study, nearly three-fifths of patients had rectal hyposensitivity.

We have been using the same technique for BET since 2012, and the present study highlights the efficacy of BET using our method. We compared BET results with those of ARM because both are physiological tests and hence are comparable. It is important to note that standardisation of BET methodology is important not only for diagnosis but for treatment as well [22]. Indian studies have noted that, despite limited availability [23], biofeedback therapy improves symptoms and BET in three-fifths of cases [24]. We noted that the combination of DRE and BET seems to be better than BET alone. However, the pickup rate of DRE is dependent on the skill of the examiner.

The present study had a large sample size and was performed by a group practicing uniform methods of DRE and BET over the past several years. It highlights the utility of BET in an Indian setting for the first time.

Despite this, there are a few limitations. It was a retrospective analysis and prone to referral bias. Tests of anorectal morphology, such as defecography, and physiology, such as electromyography and colonic transit study, have not been performed. Symptoms of other functional gastrointestinal disorders like dyspepsia, bloating, and reflux were not recorded. In the ROME III classification, only patients with FC, but not IBS-C, were eligible to be diagnosed as having a defecatory disorder. However, this has been modified as per Rome IV and may be a source of bias in the present study. Moreover, all tests of anorectal function are an imperfect simulation of the act of defecation due to the influence of laboratory conditions.

\section{Conflict of interest}

The authors declare no conflict of interest.

\section{References}

1. Jain M. Digital rectal examination - a reliable screening tool for dyssynergic defecation. Indian J Gastroenterol 2018; 37: 176-7.

2. Preston DM, Lennard-Jones JE. Anismus in chronic constipation. Dig Dis Sci 1985; 30: 413-8.

3. Noelting J, Ratuapli SK, Bharucha AE, et al. Normal values for high-resolution anorectal manometry in healthy women: effects of age and significance of rectoanal gradient. Am J Gastroenterol 2012; 107: 1530-6.

4. Ghoshal UC. Chronic constipation in Rome IV era: the Indian perspective. Indian J Gastroenterol 2017; 36: 163-73.

5. Shah N, Baijal R, Kumar P, et al. Clinical and investigative assessment of constipation: a study from a referral center in western India. Indian J Gastroenterol 2014; 33: 530-6.

6. Ratuapli S, Bharucha AE, Harvey D, Zinsmeister AR. Comparison of rectal balloon expulsion test in seated and left lateral positions. Neurogastroenterol Motil 2013; 25: e813-20.

7. Tantiphlachiva K, Rao P, Attaluri A, Rao SSC. Digital rectal examination is a useful tool for identifying patients with dyssynergia. Clin Gastroenterol Hepatol 2010; 8: 955-60.

8. Soh JS, Lee HJ, Jung KW, et al. The diagnostic value of a digital rectal examination compared with high-resolution anorectal manometry in patients with chronic constipation and fecal incontinence. Am J Gastroenterol 2015; 110: 1197-204.

9. Caetano AC, Santa-Cruz A, Rolanda C. Digital rectal examination and balloon expulsion test in the study of defecatory dis- 
orders: are they suitable as screening or excluding tests? Can J Gastroenterol Hepatol 2016; 2016: 8654314.

10. Bordeianou L, Savitt L, Dursun A. Measurements of pelvic floor dyssynergia: which test result matters? Dis Colon Rectum 2011; 54: 60-5.

11. Raza N, Bielefeldt K. Discriminative value of anorectal manometry in clinical practice. Dig Dis Sci 2009; 54: 2503-11.

12. Mibu R, Hotokezaka M, Kai T, et al. A simplified defaecographic procedure for the assessment of faecal incontinence or obstructed defaecation. Colorectal Dis 2001; 3: 328-33.

13. Glia A, Lindberg G, Nilsson LH, et al. Constipation assessed on the basis of colorectal physiology. Scand J Gastroenterol 1998; 33: 1273-9.

14. Halligan S, Thomas J, Bartram C. Intrarectal pressures and balloon expulsion related to evacuation proctography. Gut 1995; 37: 100-4.

15. Minguez M, Herreros B, Sanchiz V, et al. Predictive value of the balloon expulsion test for excluding the diagnosis of pelvic floor dyssynergia in constipation. Gastroenterology 2004; 126: 57-62.

16. Rao SSC, Welcher KD, Leistikow JS. Obstructive defecation: a failure of rectoanal coordination. Am J Gastroenterol 1998; 93: 1042-50.

17. Posserud I, Syrous A, Lindstrom L, et al. Altered rectal perception in irritable bowel syndrome is associated with symptom severity. Gastroenterology 2007; 133: 1113-23.

18. Burgell RE, Scott SM. Rectal hyposensitivity. J Neurogastroenterol Motil 2012; 18: 373-84.

19. Rao SS, Patcharatrakul T. Diagnosis and treatment of dyssynergic defecation. J Neurogastroenterol Motil 2016; 22: 423-35.

20. Rao SSC, Bharucha AE, Chiarioni G, et al. Functional anorectal disorders. Gastroenterology 2016; 150: 1430-42.

21. Rao SS, Welcher KD, Leistikow JS. Obstructive defecation: a failure of rectoanal coordination. Am J Gastroenterol 1998; 93: 1042-50.

22. Lestàr B, Penninckx F, Kerremans R. Biofeedback defaecation training for anismus. Int J Colorectal Dis 1991; 6: 202-7.

23. Jain M, Baijal R. Biofeedback therapy - challenges in Indian setting. Indian J Gastroenterol 2017; 36: 160.

24. Verma A, Misra A, Ghoshal UC. Effect of biofeedback therapy on anorectal physiological parameters among patients with fecal evacuation disorder. Indian J Gastroenterol 2017; 36: 99104.

Received: 31.05 .2019

Accepted: 31.07.2019 